\title{
Acknowledgement to Reviewers of Veterinary Sciences in 2016
}

\author{
Veterinary Sciences Editorial Office \\ Published: 10 January 2017 \\ MDPI AG, St. Alban-Anlage 66, 4052 Basel, Switzerland; vetsci@mdpi.com
}

The editors of Veterinary Sciences would like to express their sincere gratitude to the following reviewers for assessing manuscripts in 2016.

We greatly appreciate the contribution of expert reviewers, which is crucial to the journal's editorial process. We aim to recognize reviewer contributions through several mechanisms, of which the annual publication of reviewer names is one. Reviewers receive a voucher entitling them to a discount on their next MDPI publication and can download a certificate of recognition directly from our submission system. Additionally, reviewers can sign up to the service Publons (https://publons.com) to receive recognition. Of course, in these initiatives we are careful not to compromise reviewer confidentiality. Many reviewers see their work as a voluntary and often unseen part of their role as researchers. We are grateful to the time reviewers donate to our journals and the contribution they make.

If you are interested in becoming a reviewer for Veterinary Sciences, see the link at the bottom of the webpage http://www.mdpi.com/reviewers.

The following reviewed for Veterinary Sciences in 2016:

Ackermann, Mathias
Atkins, Gregory J.
Aurich, Christine
Aurich, Joerg
Baeumer, Wolfgang
Bamford, Connor
Barbosa, Angela
Beineke, Andreas
Bielefeldt-ohmann, Helle
Bionaz, Massimo
Bloom, Paul
Boswood, Adrian
Bowman, Dwight
Bown, Kevin
Bradley, Walter G.
Brisken, Cathrin
Butt, Grant
Caires, Kyle
Campaile, Floriana
Carlyon, Jason A.
Chalker, Victoria
Chapwanya, Aspinas
Chowdhury, Shafiqul
Cui, Longzhu
Cutler, Sally Jane

\author{
Gilbert, Robert O. \\ Gilk, Stacey \\ Gostelow, Ruth \\ Groban, Leanne \\ Günther, Sebastian \\ Harkin, Kenneth R. \\ Harrison, Rene \\ Harrus, Shimon \\ Heinzen, Robert \\ Hendricks, Joan C. \\ Herrel, Anthony \\ Hughes, Kate \\ Hulme-Moir, Lisa \\ Ishita, Chatterjee \\ Juarez, Michelle \\ Kim, Yunjeong \\ Korou, Laskarina-Maria \\ Kozjak-Pavlovic, Vera \\ Kurtti, Timothy \\ Leibman, Nicole \\ Leroy, Baptiste \\ Lewitus, Alan J. \\ Little, Susan E. \\ Liu, Jinghua \\ Macaluso, Kevin
}

\author{
Navas De Solis, Cristobal \\ Nieto, Nathan \\ Nuttall, Patricia A \\ Ödman, Anna \\ Pancotto, Theresa \\ Petrovski, Kiro \\ Poli, Alessandro \\ Pondeville, Emilie \\ Popov, Vsevolod Leonidovich \\ Raffe, Marc R. \\ Rego, Ryan \\ Renault, Andrew \\ Ressel, Lorenzo \\ Reusch, Claudia \\ Ricklin, Daniel \\ Risley-Curtiss, Christina \\ Rodriguez-Valle, Manuel \\ Rufini, Alessandro \\ Sassera, Davide \\ Schnittger, Leonhard \\ Shoshan, Maria \\ Siddle, Hannah V. \\ Sithole, Fortune \\ Skinner, Pamela J. \\ Song, Chang-Seon
}


Davis, Meghan Frost

Denis, Oliver

Ebo, Didier

Falconer, Ian

Fan, Timothy M.

Fassati, Ariberto

Ferguson, Sylvia

Ferreira, Fernando

Fiorito, Filomena

Fletcher, Jon

Fogle, Jonathan

Fox, Larry

Gabler, Christoph

Ghosh, Anuradha
MacNeill, Amy

Maddox, Carol

Madison-Antenucci, Susan

Mani, Rinosh

Maniscalco, Lorella

Matsuda, Hiroshi

Mergia, Ayalew

Mexas, Angela Marie

Mueller, Kristina

Mulenga, Albert

Murphy, Brian

Murugaiyan, Jayaseelan

Nakashiro, Koh-Ichi

Narasimhan, Sukanya
Sparks, Cathryn K.

Suravajhala, Prashanth

Szalay, Aladar A.

Tacke, Sabine

Taylor, Travis

Teglas, Mike

Tortonese, Domingo

Van De Walle, Gerlinde

Ventura, Nicole

Verbalis, Joseph G.

Vieths, Stefan

Wahlstrom, Helene

Yager, Eric

Zandvliet, M.M.J.M. (Maurice)

(C) 2017 by the author; licensee MDPI, Basel, Switzerland. This article is an open access article distributed under the terms and conditions of the Creative Commons Attribution (CC-BY) license (http://creativecommons.org/licenses/by/4.0/). 